\title{
Peritoneal dialysis-related peritonitis: challenges and solutions
}

\author{
William L Salzer \\ School of Medicine, University of \\ Missouri, Columbia, MO, USA
}

This article was published in the following Dove Press journal: International Journal of Nephrology and Renovascular Disease

\begin{abstract}
Peritoneal dialysis is an effective treatment modality for patients with end-stage renal disease. The relative use of peritoneal dialysis versus hemodialysis varies widely by country. Data from a 2004 survey reports the percentage of patients with end-stage renal disease treated with peritoneal dialysis to be $5 \%-10 \%$ in economically developed regions like the US and Western Europe to as much as $75 \%$ in Mexico. This disparity is probably related to the availability and access to hemodialysis, or in some cases patient preference for peritoneal over hemodialysis. Peritoneal dialysis-related peritonitis remains the major complication and primary challenge to the long-term success of peritoneal dialysis. Fifty years ago, with the advent of the Tenckhoff catheter, patients averaged six episodes of peritonitis per year on peritoneal dialysis. In 2016, the International Society for Peritoneal Dialysis proposed a benchmark of 0.5 episodes of peritonitis per year or one episode every 2 years. Despite the marked reduction in peritonitis over time, peritonitis for the individual patient is problematic. The mortality for an episode of peritonitis is $5 \%$ and is a cofactor for mortality in another $16 \%$ of affected patients. Prevention of peritonitis and prompt and appropriate management of peritonitis is essential for the longterm success of peritoneal dialysis in all patients. In this review, challenges and solutions are addressed regarding the pathogenesis, clinical features, diagnosis, treatment, and prevention of peritoneal dialysis-related peritonitis from the viewpoint of an infectious disease physician.
\end{abstract} Keywords: peritoneal dialysis, related peritonitis

\section{Introduction}

The use of peritoneal dialysis (PD) for renal replacement therapy varies widely in different regions of the world and in individual countries. ${ }^{1}$ In economically developed countries, the choice of PD versus hemodialysis is sometimes a matter of patient preference and sometimes due to lack of a hemodialysis unit that is easily accessible to the patient's home. In less economically privileged areas, PD may be the first choice, due to higher costs and difficulty accessing a hemodialysis unit. In this paper, the pathogenesis, clinical features, diagnosis, and therapy of the commonest microorganisms causing PD peritonitis are reviewed, as well as measures to reduce the occurrence of peritonitis.

\section{PD-related peritonitis - pathogenesis}

The PD catheter is the source of infection for the vast majority of PD-related cases of peritonitis. The catheter provides a portal of entry for organisms into the normally sterile peritoneum. Most cases of PD-related peritonitis are the result of "touch 
contamination", where the patient or their helper inadvertently breaks sterile technique and contaminates the catheter or its connections. The most common pathogens are coagulase-negative staphylococcal species (eg, Staphylococcus epidermidis) that commonly colonize human skin and hands, and Staphylococcus aureus, which together are responsible for $50 \%$ or more of infections in most series. ${ }^{2-4}$ Exit-site and tunnel infections may also lead to peritonitis. Less commonly, the abdomen itself may be the source. Diverticulitis, appendicitis, cholecystitis, or a perforated viscus may be the source, as well as intra-abdominal surgery, colonoscopy, hysteroscopy, and transmigration of bowel flora from constipation. In cases with an intra-abdominal source, the infecting organisms are usually Gram-negative enteric bacteria, streptococci and anaerobic bacteria. Bacteremia from another source may also seed the peritoneum.

Upon entry to the peritoneum, microorganisms find a very hospitable environment. It is warm, dark, and there are lots of nutrients like glucose. Also, in the uninflamed peritoneum, there is very little host defense, with a small number of peritoneal macrophages, and very few host-defense proteins like immunoglobulins or complement. In this environment, organisms can rapidly proliferate unimpeded. Within hours, bacterial products, cell-wall components for Gram-positive and endotoxin for Gram-negative bacteria induce an inflammatory response. Polymorphonuclear leukocytes (PMNLs) and activated macrophages enter the peritoneum and induce inflammation, which is manifested as abdominal pain, fever, peripheral blood leukocytosis, and cloudy dialysate from the increased number of cells in the peritoneal fluid.

\section{Exit-site and tunnel infections}

Most cases of PD-related peritonitis are the result of touch contamination, in which the infecting organism gains access to the peritoneum via the catheter lumen. A small percentage result from exit-site or tunnel infections, in which organisms spread down the catheter tunnel outside the lumen to the peritoneum. ${ }^{3}$ Exit-site infection presents as purulent drainage at the exit site, with or without erythema. Erythema by itself does not indicate an exit-site infection. ${ }^{5}$ Swelling, induration, and/or erythema that extends more than $2 \mathrm{~cm}$ proximally to the exit site defines a tunnel infection, which presents a much higher risk of developing peritonitis. The organisms causing exit-site infections with the highest risk of subsequent peritonitis are $S$. aureus, coagulase-negative staphylococci, diphtheroids, streptococcal species, Pseudomonas aeruginosa, and Candida. ${ }^{6}$ Nontuberculous mycobacteria (NTM) species have also been reported to cause exit-site infections and peritonitis, particularly in patients using gentamicin cream for exit-site prophylaxis. ${ }^{7}$

Purulent drainage from the exit site should be sent for Gram staining; however, cultures should not be obtained if the exit site is erythematous without exudate, because cultures will usually grow an organism that is just colonizing and the patient will often be unnecessarily exposed to antibiotics, fostering the emergence of drug-resistant bacteria. Exit-site infections can usually be treated with oral antibiotics for 2-3 weeks, guided by drug-susceptibility results. See Table 1 for suggested dosing regimens for patients on PD. Exit-site infections that are refractory to antibiotics will usually require catheter removal and replacement at another site. ${ }^{2}$

Tunnel infections are more serious, and pose a greater risk of catheter loss and peritonitis. Usually, there is purulent or serosanguinous drainage from the exit site, along with swelling, erythema, induration, and pain along the catheter tunnel. The most common organisms causing tunnel infection are $S$. aureus and $P$. aeruginosa, often preceded by an exit-site infection. Tunnel infections require treatment with systemic antibiotics, guided by culture and sensitivity reports. In most

Table I Systemic antibiotic-dosing recommendations for the treatment of peritonitis ${ }^{2}$

\begin{tabular}{|c|c|}
\hline Drug & Dosing \\
\hline \multicolumn{2}{|l|}{ Antibacterials } \\
\hline Ciprofloxacin (237) & oral $250 \mathrm{mg} \mathrm{BD}$ \\
\hline \multirow[t]{2}{*}{ Colistin (288) } & IV $300 \mathrm{mg}$ loading, then \\
\hline & I50-200 mg daily \\
\hline Ertapenem (289) & IV 500 mg daily \\
\hline Levofloxacin (239) & oral $250 \mathrm{mg}$ daily \\
\hline Linezolid (290-292) & IV or oral $600 \mathrm{mg} \mathrm{BD}$ \\
\hline Moxifloxacin (293) & oral $400 \mathrm{mg}$ daily \\
\hline \multirow[t]{2}{*}{ Rifampicin $(294,295)$} & 450 mg daily for $B W<50$ kg; \\
\hline & $600 \mathrm{mg}$ daily for $\mathrm{BW} \geq 50 \mathrm{~kg}$ \\
\hline Trimethoprim/ & oral $160 \mathrm{mg} / 800 \mathrm{mg} \mathrm{BD}$ \\
\hline \multicolumn{2}{|l|}{ Sulfamethoxazole (252) } \\
\hline \multicolumn{2}{|l|}{ Antifungals } \\
\hline \multirow[t]{4}{*}{ Amphotericin (296) } & IV test dose I mg; starting dose \\
\hline & $0.1 \mathrm{mg} / \mathrm{kg} /$ day over 6 hours; \\
\hline & increased to target dose \\
\hline & $0.75-1.0 \mathrm{mg} / \mathrm{kg} /$ day over 4 days \\
\hline Caspofungin $(297,298)$ & IV $70 \mathrm{mg}$ Loading, then $50 \mathrm{mg}$ daily \\
\hline \multirow[t]{2}{*}{ Fluconazole (299) } & oral $200 \mathrm{mg}$ loading, then \\
\hline & $50-100 \mathrm{mg}$ daily \\
\hline Flucytosine (296) & oral I g/day \\
\hline Posaconazole (300) & IV $400 \mathrm{mg}$ every 12 hours \\
\hline Voriconazole (30I-303) & oral $200 \mathrm{mg}$ every 12 hours \\
\hline
\end{tabular}

Notes: a Ciprofloxacin $500 \mathrm{mg}$ BID may be needed if residual glomerular filtration rate is above $5 \mathrm{~mL} / \mathrm{min}$; bexpressed as colistin base activity. Reproduced from $\mathrm{Li}$ PK, Szeto CC, Piraino B, et al. ISPD peritonitis recommendations: 2016 update on prevention and treatment. Perit Dial Int. 2016;36:48I-508, ${ }^{2}$ with permission from Peritoneal Dialysis International.

Abbreviations: BID, bis in die (twice daily); IV, intravenous; BW, body weight. 
cases, antibiotic therapy alone is ineffective and catheter removal will be required, with subsequent catheter replacement. In tunnel infections that have progressed to peritonitis, at least 2 weeks of effective antibiotic therapy after catheter removal is recommended before catheter replacement. ${ }^{6}$

\section{PD-related peritonitis - diagnosis}

During their PD, patients must be trained to be alert for the signs and symptoms of peritonitis. New onset of abdominal pain, fever, or the appearance of cloudy effluent should prompt immediate notification of their dialysis provider for urgent evaluation. In patients receiving automated dialysis, the first drain is the most likely to be cloudy and have an elevated white blood cell (WBC) count. If the patient presents with a dry abdomen, $1 \mathrm{~L}$ dialysate should be infused and allowed to dwell for 2 hours before sampling. Ideally, samples of dialysis effluent should be obtained for WBC count, differential, Gram stain, and culture before the administration of antibiotics. If immediate access to a facility is not possible, due to travel or distance, a sample of dialysate should be obtained under sterile conditions and refrigerated if antibiotics will be administered prior to formal sampling. Prompt fluid analysis and initiation of empiric antibiotic therapy improves outcomes. WBC count and differential should be performed on dialysis effluent. The presence of $>100 \mathrm{WBCs} / \mu \mathrm{L}$ with $>50 \%$ neutrophils on a 2 -hour dwell sample usually indicates peritonitis. ${ }^{2}$

Culture with identification of the infecting organism and determination of its antimicrobial-drug susceptibility is essential for focused antimicrobial therapy. Ideally, peritoneal fluid should be inoculated into blood-culture media at the bedside. Direct inoculation into BACTEC/Alert culture bottles at the bedside has been shown to increase yield and reduce culture-negative cases. ${ }^{8}$ Obtaining a $50 \mathrm{~mL}$ sample of peritoneal fluid effluent, centrifuging at $3,000 \mathrm{~g}$ for $15 \mathrm{~min}$ utes, and resuspending the pellet in 3-5 $\mathrm{mL}$ buffer followed by Gram staining and culture of the resuspended pellet can increase the yield of cultures and Gram stains. A positive Gram stain will facilitate more focused initial antimicrobial therapy directed at Gram-positive or Gram-negative bacteria or yeast. In most cases, however, fluid Gram stains are negative. Bacterial cultures generally take 1-3 days to yield an organism. Identification of an organism and testing for drug susceptibility allows focused antimicrobial therapy.

\section{Challenges in diagnosis}

Unfortunately, PD-fluid cultures do not always yield an organism in patients with clinical findings of peritonitis.
International Society for Peritoneal Dialysis (ISPD) guidelines recommend a benchmark of $<20 \%$ culture-negative cases. The most common cause of culture-negative cases is initiation of antibiotics before cultures are obtained. Patients who present with clinical peritonitis, have negative peritoneal cultures, and who improve on empiric therapy with decreased symptoms and falling peritoneal WBC counts usually have infection with susceptible Gram-positive bacteria and can be treated for 2 weeks. ${ }^{2}$ Other causes of culture-negative cases are infection with fastidious bacterial organisms, acid-fast bacilli (AFB), or fungal organisms. In these cases, if clinical improvement in symptoms and peritoneal WBC count has not occurred after 3 days of empiric antibiotic therapy and initial peritoneal fluid cultures remain negative after 3 days, the initial peritoneal fluid-culture bottles should be subcultured onto solid media and enriched liquid media like thioglycolate. Also, fluid should be obtained and cultured for fungi and AFB.

\section{Future solutions in diagnosis}

In the initial evaluation of peritonitis, Gram stains of the peritoneal fluid are usually negative. It usually takes $1-3$ days for the fluid cultures to become positive and another 2 days for identification of the species and results of antimicrobial-susceptibility testing. There now exist a number of commercially available multiplex polymerase chain reaction (PCR) panels for respiratory, gastrointestinal (GI) and central nervous system pathogens that yield a species ID result in an hour on direct clinical specimens. In addition, matrix-assisted laser desorption/ionization time of flight (MALDITOF) performed on positive cultures can provide species identification in less than an hour, ${ }^{9}$ and real-time PCR systems can provide species identification and common drug-resistance data like detection of methicillin-resistant $S$. aureus (MRSA) and vancomycinresistant Enterococcus (VRE) on positive cultures.

\section{Initial empiric antibiotic therapy for peritonitis}

After obtaining PD fluid for microbiologic testing, empiric antibiotic therapy should be started as soon as possible. In the absence of a positive Gram stain, empiric therapy should include antibiotics that cover both Gram-negative and Gram-positive bacteria. The choice of antibiotics should be determined by the prevalence and types of antibioticresistant bacterial isolates from peritonitis patients in the program. Antibiotics should be administered intraperitoneally in most cases. ${ }^{2}$ For empiric coverage of Gram-positive bacteria, recommended drugs are cefazolin or vancomycin. In 
programs with a low incidence of MRSA species and enterococci, cefazolin would be preferred. In many programs, MR coagulase-negative staphylococci and MRSA are common pathogens in peritonitis patients, and vancomycin should be used initially, pending culture results. For empiric coverage of Gram-negative bacteria, initial therapy should include a third-generation cephalosporin (ceftazidime or cefepime) or an aminoglycoside (gentamicin, tobramycin, or amikacin). Again, the choice of antibiotics for empiric Gram-negative coverage is dependent on the species of bacteria and the prevalence and types of antibiotic-resistant pathogens in the program.

\section{Definitive therapy for PD-related peritonitis}

In most cases, culture and susceptibility results will be available in 3-5 days, and antibiotic therapy can be focused on a single agent. In culture-negative cases, particularly those that are not severe, the most likely pathogen is a Gram-positive organism, most often a coagulase-negative Staphylococcus sp., and Gram-negative coverage can be discontinued. If the patient has not responded clinically after 3-5 days, as described earlier, repeat peritoneal fluid sample should be cultured for unusual pathogens.

\section{Antibiotic administration for PD peritonitis}

The preferred method of antibiotic administration for PDrelated peritonitis is intraperitoneal. The advantages of intraperitoneally administered antibiotics include delivery of a high concentration of antibiotics at the site of infection, antibiotics also being absorbed into the systemic circulation and diffusing back into the peritoneum, allowing daily or less frequent administration, and intravenous (IV) access, which would be required for $2-4$ weeks, is not necessary (Table 2).

\section{Challenges}

The 2016 ISPD guidelines express concerns about the effectiveness of once-daily intraperitoneal dosing of cephalosporins in patients on APD, and recommend including antibiotics in each bag of dialysate for patients on automated PD or alternatively converting to continuous ambulatory PD for the duration of therapy. This would be quite cumbersome for patients dialyzing at home. However, there have been two published series that examined just adding antibiotics to their "day dwell" in patients on automated PD, and found comparable success rates compared to patients using antibiotics in each bag on the cycler. ${ }^{10,11}$

\section{PD-related peritonitis: clinical features, diagnosis, and therapy by organism \\ Coagulase-negative staphylococci}

S. epidermidis is the most frequently identified cause of PD-associated peritonitis. While $S$. epidermidis is the most common of the coagulase-negative staphylococci, there are at least 40 other species of coagulase-negative Staphylococcus that have been reported as a cause of human infections. These organisms are the predominant normal flora on human skin and generally low-virulence pathogens, but are among the most common causes of device-related infections, because of their ability to adhere to nonbiologic surfaces and produce biofilms. ${ }^{12}$ In a biofilm, microorganisms are embedded in a glycocalyx, analogous to a coral reef in that the organisms on the outer surface are metabolically active and dividing, while the ones deep in the biofilm are essentially dormant. ${ }^{13}$ Antibiotics work only on actively dividing microorganisms, such that the organisms deep in the biofilm are not affected, resulting in a nidus for relapse. Relapsing or recurrent peritonitis is often seen in cases of coagulase-negative Staphylococcus peritonitis. ${ }^{14}$ Relapsing peritonitis usually requires catheter removal followed by replacement.

\section{An illustrative case}

A 65-year-old man on PD presents with a 6-hour history of abdominal pain, fever, and cloudy dialysate. He relates that he might have touched his connector without gloves on yesterday. His temperature is $38^{\circ} \mathrm{C}$, and he has mildmoderate abdominal pain. Peritoneal fluid analysis reveals WBC $1,400 / \mu \mathrm{L}$, with $85 \%$ PMNLs, and fluid Gram stain reveals PMNLs, but no organisms. Cultures are obtained, and he is started empirically on intraperitoneal vancomycin and ceftazidime. By day 3, he has symptomatically improved, with clearing of his effluent. Fluid cultures grow coagulasenegative Staphylococcus, which is sensitive to cefazolin and vancomycin. Vancomycin and ceftazidime are discontinued, and he is treated with intraperitoneal cefazolin for 14 days. His peritonitis resolves without a recurrence.

Clinically, peritonitis caused by coagulase-negative Staphylococcus usually presents with relatively mild signs and symptoms compared to more virulent organisms. ${ }^{15}$ The incidence of MR isolates has been increasing, reported in $70 \%$ of coagulase-negative Staphylococcus isolates in a series from Brazil. ${ }^{16}$ With appropriate intraperitoneal antibiotic therapy, clinical improvement is usually apparent by day 3. Treatment for 14 days is recommended, with cure 
Table 2 IP antibiotic-dosing recommendations for the treatment of peritonitis ${ }^{2}$

\begin{tabular}{|c|c|c|}
\hline & Intermittent (one exchange daily) & Continuous (all exchanges) \\
\hline \multicolumn{3}{|l|}{ Aminoglycosides } \\
\hline Amikacin & 2 mg/kg daily (252) & LD 25 mg/L, MD 12 mg/L (253) \\
\hline Gentamicin & 0.6 mg/kg daily (254) & LD 8 mg/L, MD 4 mg/L $(255,256)$ \\
\hline Netilmicin & $0.6 \mathrm{mg} / \mathrm{kg}$ daily $(233)$ & MD $10 \mathrm{mg} / \mathrm{L}(257)$ \\
\hline Tobramycin & 0.6 mg/kg daily (253) & LD 3 mg/kg, MD 0.3 mg/kg $(258,259)$ \\
\hline \multicolumn{3}{|l|}{ Cephalosporins } \\
\hline Cefazolin & I5-20 mg/kg daily $(260,26 \mathrm{I})$ & LD 500 mg/L, MD I 25 mg/L (254) \\
\hline Cefepime & I,000 mg daily $(262,263)$ & LD $250-500$ mg/L, MD $100-125$ mg/L $(262,263)$ \\
\hline Cefoperazone & no data & LD 500 mg/L, MD 62.5-I25 mg/L $(264,265)$ \\
\hline Cefotaxime & 500-I,000 mg daily (266) & no data \\
\hline Ceftazidime & $\mathrm{I}, 000-\mathrm{I}, 500 \mathrm{mg}$ daily $(267,268)$ & LD 500 mg/L, MD 125 mg/L (236) \\
\hline Ceftriaxone & I,000 mg daily (269) & no data \\
\hline \multicolumn{3}{|l|}{ Penicillins } \\
\hline Penicillin G & no data & LD 50,000 unit/L, MD 25,000 unit/L (270) \\
\hline Amoxicillin & no data & MD I50 mg/L (27I) \\
\hline Ampicillin & no data & MD I 25 mg/L $(272,273)$ \\
\hline Ampicillin/Sulbactam & 2 g/I g every 12 hours (274) & LD 750-100 mg/L, MD 100 mg/L (253) \\
\hline Piperacillin/Tazobactam & no data & LD 4 g/0.5 g, MD I g/0.I 25 g (275) \\
\hline \multicolumn{3}{|l|}{ Others } \\
\hline Aztreonam & 2 g daily (242) & LD I,000 mg/L, MD 250 mg/L $(243,244)$ \\
\hline Ciprofloxacin & no data & MD $50 \mathrm{mg} / \mathrm{L}(276)$ \\
\hline Clindamycin & no data & MD 600 mg/bag (277) \\
\hline Daptomycin & no data & LD 100 mg/L, MD 20 mg/L (278) \\
\hline Imipenem/Cilastatin & $500 \mathrm{mg}$ in alternate exchange (244) & LD 250 mg/L, MD 50 mg/L (236) \\
\hline Ofloxacin & no data & LD 200 mg, MD 25 mg/L (279) \\
\hline Polymyxin B & no data & MD 300,000 unit (30 mg)/bag (280) \\
\hline Quinupristin/Dalfopristin & $25 \mathrm{mg} /$ Lin alternate exchange ${ }^{\mathrm{a}}(28 \mathrm{I})$ & no data \\
\hline Meropenem & I g daily (282) & no data \\
\hline Teicoplanin & 15 mg/kg every 5 days (283) & LD 400 mg/bag, MD 20 mg/bag (229) \\
\hline Vancomycin & I5-30 mg/kg every 5-7 days ${ }^{\mathrm{b}}(284)$ & LD 30 mg/kg, MD 1.5 mg/kg/bag (285) \\
\hline \multicolumn{3}{|l|}{ Antifungals } \\
\hline Fluconazole & IP 200 mg every $24-48$ hours (286) & no data \\
\hline Voriconazole & IP 2.5 mg/kg daily (287) & no data \\
\hline
\end{tabular}

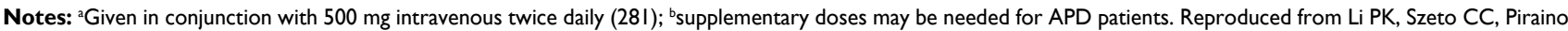
B, et al. ISPD peritonitis recommendations: 2016 update on prevention and treatment. Perit Dial Int. 2016;36:48I-508, ${ }^{2}$ with permission from Peritoneal Dialysis International. Abbreviations: LD, loading dose; MD, maintenance dose; IP, intraperitoneal; APD, automated peritoneal dialysis.

rates about $70 \%$ or more in most series. ${ }^{16}$ Catheter replacement or removal is often required for relapsing or recurrent cases, which presumably occurs due to biofilm formation in the catheter.

\section{S. aureus peritonitis}

$S$. aureus generally produces a more clinically severe form of peritonitis in PD patients, because of its many virulence factors. $S$. aureus colonizes many patients in their nares and on their skin, as well as disruptions of the skin, like an exit site. In one study, $45 \%$ of patients initiating PD were found to be nasal carriers of $S$. aureus and had a significantly higher risk of developing an exit-site infection. ${ }^{17} \mathrm{~S}$. aureus is a common cause of exit-site and tunnel infections. Mupirocin for exit-site prophylaxis seems to reduce exit-site infections, peritonitis, and catheter loss due to $S$. aureus. ${ }^{18}$
An illustrative case: MRSA tunnel infection leading to peritonitis

A 55-year-old man presents with pain and swelling along the tunnel of his peritoneal catheter. He is afebrile and treated empirically with IV vancomycin and piperacillin-tazobactam. He undergoes incision and drainage of his catheter tunnel. Gram staining of the tunnel exudate reveals many PMNLs and Gram-positive cocci in clusters and growing MRSA. Piperacillin-tazobactam is discontinued, and he is treated with vancomycin. He is taken to the OR, where the presternal portion of his catheter is externalized. After 5 days of vancomycin therapy, he is discharged on oral clindamycin.

Five weeks later, he returns with fever, chills, diffuse abdominal pain, and purulent drainage from his exit site, and has noticed cloudy, bloody effluent. His temperature is $38.1^{\circ} \mathrm{C}$, his bowel sounds are decreased, and there is diffuse 
abdominal tenderness with rebound tenderness. Peritoneal fluid is cloudy and reddish in color, with 33,800 WBCs and 92\% PMNLs. Cultures of blood, peritoneal fluid, and exit site all grow MRSA. A transesophageal echocardiogram is negative for vegetations. His peritoneal catheter is removed, he transfers to hemodialysis, and is treated with IV vancomycin for 3 weeks after catheter removal.

\section{Comment}

Tunnel infection with tenderness, swelling, and redness extending $>2 \mathrm{~cm}$ proximal to the exit site is very difficult to resolve with antibiotic therapy alone. In this case, in retrospect, the catheter should have been removed and replaced at the initial presentation.

As this case illustrates, peritonitis caused by $S$. aureus is usually clinically severe. ${ }^{19,20}$ It can occur after touch contamination or via tunnel or exit-site infection. Treatment depends on the antimicrobial susceptibility of the infecting strain. For methicillin-susceptible $S$. aureus, cefazolin intraperitoneally is the drug of choice, while for MRSA intraperitoneal vancomycin is the first choice. If the patient is allergic to vancomycin or has a vancomycin-resistant strain (VR), the lipopeptide daptomycin can be used. ${ }^{21-23}$ A variety of dosing regimens for daptomycin have been reported, but the best regimen is probably $200 \mathrm{mg}$ intraperitoneally with a 6-hour dwell once a day. ${ }^{23}$ Linezolid has good activity against MRSA, and has been used successfully to treat VRE peritonitis. The dose used has been $600 \mathrm{mg}$ IV twice daily, ${ }^{24}$ but the oral bioavailability is such that oral dosing should be just as effective. However, $600 \mathrm{mg}$ twice daily has been associated with hematologic toxicity and lactic acidosis in patients with $\mathrm{ESRD},{ }^{25}$ and a dose of $400 \mathrm{mg}$ twice daily is effective and safer. Ceftaroline is a relatively new third-generation cephalosporin that is unique among $\beta$-lactam antibiotics in that it is very active against MRSA. ${ }^{26}$ Ceftaroline also has Gram-positive activity comparable to cefazolin and a Gram-negative spectrum similar to cefotaxime. Ceftaroline might be an effective alternative to vancomycin for MRSA peritonitis, but to my knowledge has not been studied. There is some evidence that adding rifampin for 5-7 days to primary antistaphylococcal drugs reduces relapses and recurrent peritonitis. ${ }^{19}$ However, rifampin is a potent inducer of drug-metabolizing enzymes, which may reduce the concentrations of other medications.

$S$. aureus peritonitis should be treated for 3 weeks with antibiotics. Prompt catheter removal is necessary in patients with refractory or relapsing peritonitis and for exit-site and tunnel infections that fail to respond to antibiotic therapy. Peritonitis caused by $S$. aureus has relatively poor outcomes, with $20 \%$ relapsing, $23 \%$ requiring catheter removal, $18 \%$ transferring to hemodialysis, and $2 \%$ mortality. ${ }^{20,21}$

\section{Streptococcal peritonitis}

Streptococcal peritonitis is less common, accounting for $5 \%-10 \%$ of cases in most series. ${ }^{27,28}$ Peritonitis caused by streptococcal species resembles that caused by staphylococcal species in that the clinical features depend on the infecting species. Peritonitis caused by $\beta$-hemolytic streptococci groups A, B, and others, presents like $S$. aureus, with more inflammatory peritoneal fluid parameters, more pain, and often with a more septic picture. Viridans streptococci are a large group of species, defined by $\alpha$-hemolysis on blood agar cultures. Like coagulase-negative staphylococci, most viridans streptococci isolates are not very virulent organisms, being normal inhabitants of the mouth and GI tract. Cases of viridans streptococcus peritonitis have been reported after dental work. ${ }^{28}$

$\beta$-Hemolytic streptococci are all very sensitive to penicillin and other $\beta$-lactam antibiotics. Viridans streptococcal species are usually sensitive to $\beta$-lactams, but there has been increasing resistance to penicillins in some areas. ${ }^{29}$ ISPD guidelines recommend intraperitoneal ampicillin for the treatment of streptococcal peritonitis. ${ }^{2}$ Ampicillin can be problematic for outpatient peritonitis therapy, because it begins to break down in solution after $6-8$ hours. ${ }^{30}$ A better choice for penicillin-susceptible stains might be cefazolin, which is stable in solution for 9 days at room temperature and 3 weeks refrigerated. ${ }^{30}$ For penicillin-resistant viridans streptococci, vancomycin would be the drug of choice. Most patients have good outcomes with 2 weeks of antibiotic therapy, with success rates of up to $90 \%$. $^{28,29,32}$ Some studies have reported an increased risk of recurrent or relapsing peritonitis with viridans streptococci peritonitis. ${ }^{28,31}$

\section{Enterococcal peritonitis}

Enterococcus spp. are normal inhabitants of the GI tract, and may colonize or infect the genitourinary (GU) tract. The most common species isolated from human infections are Enterococcus faecalis and Enterococcus faecium. E. faecalis is usually susceptible to ampicillin, penicillin, and vancomycin, but can be VR if it carries the vanB gene. E. faecium is always resistant to penicillin, ampicillin, and carbapenems, and is the most common species of enterococci that are VR when it possesses the vanA gene. All enterococcal species are inherently resistant to all cephalosporin antibiotics.

Enterococcal peritonitis probably results from touch contamination and possibly from GI sources. Single-organism 
enterococcal peritonitis is usually not too severe clinically and responds well to appropriate therapy, with successful treatment in up to $90 \%$ of cases..$^{33}$ However, in up to $45 \%$ of cases in which enterococci are isolated, other organisms are isolated as well, ${ }^{32,33}$ suggesting an intra-abdominal source of infection. Outcomes are significantly worse with polymicrobial enterococcal peritonitis, which results in higher rates of catheter removal and permanent transfer to hemodialysis. ${ }^{32}$

Antimicrobial treatment options for enterococcal peritonitis are limited, due to the inherent resistance to all $\beta$-lactams except ampicillin, penicillin, and piperacillin which are problematic for the treatment of peritonitis. ISPD guidelines recommend treatment with intraperitoneal vancomycin for 3 weeks, with the addition of gentamicin in severe cases. ${ }^{2}$ In cases of polymicrobial peritonitis, additional antimicrobial agents may be necessary, based on the drug susceptibilities of the other infecting organisms. Treatment of VR enterococcal peritonitis is challenging. VRE is usually resistant to all commonly used antibiotics. A minority of VRE infections are caused by E. faecalis, which is usually susceptible to penicillin and ampicillin. Most VRE is E. faecium, which is inherently resistant to ampicillin. For VR E. faecium peritonitis, therapeutic choices include daptomycin, linezolid, and quinupristin-dalfopristin. Among these three compounds, the most published experience is with daptomycin, which is recommended in the ISPD guidelines. ${ }^{2}$ The most frequently reported daptomycin-dosing regimen has been a loading dose of $100 \mathrm{mg} / \mathrm{L}$ intraperitoneally, followed by $20 \mathrm{mg} / \mathrm{L}$ intraperitoneally for subsequent doses. ${ }^{34,35}$ Daptomycin has also been used successfully in a patient on automated perineal dialysis given $7 \mathrm{mg} / \mathrm{kg}$ intraperitoneally every 48 hours $^{22}$ and in a patient given $5 \mathrm{mg} / \mathrm{kg}$ IV every 48 hours. ${ }^{21}$ Another simpler regimen is $200 \mathrm{mg}$ intraperitoneally during the long dwell in patients on automated PD. ${ }^{23}$ There is less published experience with linezolid, with two reports of successful treatment of VRE peritonitis treated with linezolid $600 \mathrm{mg}$ IV every 12 hours. ${ }^{24,36}$ Case series have reported hematologic toxicity with anemia, leukopenia, and thrombocytopenia in patients treated with linezolid $600 \mathrm{mg}$ twice daily for 14 days or more and one report of fatal lactic acidosis. ${ }^{25,37}$ Dosing linezolid at 400 $\mathrm{mg}$ twice daily would achieve therapeutic concentrations and be safer. Also, taken orally, linezolid is $100 \%$ bioavailable, so IV or intraperitoneal dosing is probably not necessary. ${ }^{38}$

\section{Corynebacterium peritonitis}

Corynebacterium is a genus with many species of small Grampositive rods. Often referred to as diphtheroids, they commonly colonize human skin and mucous membranes. Other than
Corynebacterium diphtheriae, they are generally low-virulence pathogens, resembling infections caused by coagulase-negative Staphylococcus. In most large series, corynebacteria account for less than $5 \%$ of PD-associated peritonitis cases. In the two largest series of corynebacterial peritonitis, one reported generally good outcomes and recommended therapy with cefazolin, ${ }^{39}$ while the other reported a recurrence rate of $48 \%$ and found better outcomes with vancomycin. ${ }^{40}$ Most species of corynebacteria are susceptible to cefazolin, but some species Corynebacterium jeikeium and Corynebacterium striatum - are multiresistant and susceptible only to vancomycin (see case). ISPD guidelines recommend treatment for 3 weeks. ${ }^{2}$

\section{An illustrative case}

A 57-year-old man presents with a 2-day history of abdominal pain and cloudy effluent. He is afebrile and has mild abdominal tenderness without rebound tenderness. His PD fluid has 2,000 WBCs with $83 \%$ PMNLs and a negative Gram stain. He receives empiric therapy with intraperitoneal vancomycin and ceftazidime. His peritoneal fluid cultures grow C. jeikeium, but our lab does not perform drug susceptibilities on corynebacteria. His therapy is changed to cefazolin alone. Unfortunately, $C$. jeikeium is always resistant to cefazolin and most other antibiotics, and an infectious-disease consultation was not requested. Three months later, he returns with abdominal pain, bloody effluent, and a poorly functioning catheter. His peritoneal fluid has 1,800 WBCs with 93\% PMNLs, 8,000 RBCs, and a negative Gram stain. He is treated empirically with intraperitoneal vancomycin and ceftazidime. Peritoneal fluid cultures again grow C. jeikeium. Peritoneal catheter removal is attempted but is unsuccessful, due to extensive adhesions, and he is switched to hemodialysis. Blood cultures are negative, but peritoneal fluid cultures again grow $C$. jeikeium. His catheter is finally removed, and the tip grows C. jeikeium. Computed tomography of his abdomen reveals $23 \times 10 \times 20 \mathrm{~cm}$ anterior abdominal fluid collection, and he is diagnosed with encapsulating peritonitis.

\section{Comment}

In this unfortunate case, it was not recognized that he was infected with a resistant species of Corynebacterium, which was inadequately treated and resulted in technique failure.

\section{Gram-negative enteric peritonitis}

Gram-negative enteric bacteria are members of the family Enterobacteriaceae and include the species Escherichia coli and Klebsiella, Enterobacter, Citrobacter, and Proteus spp. This group of organisms are part of the normal flora of the 
GI tract, may colonize the upper aerodigestive tract and GU tract, and are widespread in the environment. These organisms have a bilayer cell membrane that contains lipopolysaccharide (endotoxin), which is an important virulence factor.

In most case series of peritonitis, Gram-negative enteric bacteria account for $10 \%-25 \%$ of cases, with the highest rates reported in Asia and Australia. In recent years, the percentage of cases caused by these organisms has increased, possibly because of the relative decrease in staphylococcal infections as a result of widespread use of mupirocin for exit-site prophylaxis. ${ }^{41}$ Peritonitis probably results from touch contamination, but sometimes from an exit-site or tunnel infection or from an intra-abdominal source. Recent antibiotic therapy for peritonitis or other indications is a risk factor for Gram-negative enteric peritonitis. ${ }^{42}$

The clinical signs and symptoms of peritonitis caused by Gram-negative enteric bacteria tend to be more severe, with fever, more severe abdominal pain, nausea, vomiting, and diarrhea. ${ }^{42} \mathrm{~A}$ recent course of antibiotics for peritonitis, exit-site infection, or other reasons is present in up to a third of patients. ${ }^{42,43}$ E. coli accounts for $30 \%-50 \%$ of cases, followed by Klebsiella and Enterobacter spp. Polymicrobial Gram-negative peritonitis should raise suspicion for an intraabdominal source, like a perforated viscus or diverticulitis.

Most if not all centers include antibiotics with broad Gram-negative activity, like ceftazidime or cefepime, or gentamicin or tobramycin in initial empiric therapy. In the past 10-20 years, however, Enterobacteriaceae have become increasingly resistant to many antibiotics..$^{41,42,44,45}$ Extendedspectrum $\beta$-lactamases (ESBLs) are plasmid-mediated enzymes that break down cephalosporins. ${ }^{44}$ ESBL-producing strains of Gram-negative rods seem to be more prevalent in South Asia, with two series reporting that $12 \%$ and $35 \%$ of their E. coli peritoneal isolates produced ESBL. ${ }^{46,47}$ Patients infected with ESBL-producing strains of $E$. coli are three to four times more likely to fail treatment than patients with non-ESBL-producing strains. ${ }^{47}$ As always, definitive therapy should be based on drug-susceptibility testing, but generally ESBL-producing strains are usually susceptible to carbapenems like imipenem, meropenem, and ertapenem and usually susceptible to aminoglycosides like gentamicin or tobramycin. In addition, SPICE (Serratia, Pseudomonas, Proteus, Citrobacter, and Enterobacter spp.) organisms usually have AmpC $\beta$-lactamase enzymes, which break down most cephalosporin antibiotics and are best treated with carbapenems, fluoroquinolones, or aminoglycosides, depending on drug susceptibilities. ${ }^{43}$ Of even greater concern is the emergence and spread of carbapenem-resistant Enterobacteriaceae
(CRE) or Klebsiella pneumoniae producing carbapenemase (KPC). ${ }^{48}$ This form of antibiotic resistance is transmitted between bacterial strains on mobile genetic elements and results in resistance to all $\beta$-lactam antibiotics, including carbapenems, and usually resistant to fluoroquinolones and many aminoglycosides. Treatment options are limited in most cases to polymyxin, colistin, or tigecycline, depending on drug-susceptibility studies. ${ }^{48}$

Most empiric antibiotic regimens for PD-associated peritonitis include fairly broad coverage for Gram-negative bacteria, including broad-spectrum cephalosporins (ceftazidime or cefepime) or an aminoglycoside. Upon receipt of culture and sensitivity results, therapy can be narrowed to one effective antibiotic. Therapy should be continued for 3 weeks. ${ }^{2}$ Despite optimal antibiotic therapy, peritonitis caused by Gram-negative enteric bacteria results in relatively high failure rates, catheter removal, permanent transfer to hemodialysis, and death. ${ }^{42,43}$

\section{Peritonitis caused by nonfermenting Gram-negative rods: Pseudomonas, Acinetobacter, and Stenotrophomonas}

Pseudomonas, Acinetobacter, and Stenotrophomonas are strict aerobes (nonfermenters) that individually account for less than $5 \%$ of peritonitis cases in most programs. The importance of these organisms is that they are usually resistant to most antibiotics, making them more difficult to treat, and have high failure rates, often resulting in catheter removal.

\section{Pseudomonas peritonitis}

Pseudomonas aeruginosa is a common nosocomial pathogen that is usually multidrug-resistant. Pseudomonas peritonitis often occurs after a recent course of antibiotics for peritonitis or other indications. ${ }^{49}$ Treatment with two antibiotics for 3 weeks is recommended, ${ }^{2}$ and catheter removal is recommended if a tunnel infection is present or a suboptimal response to antibiotic therapy occurs. ${ }^{49,50}$ Definitive therapy is based on the results of antimicrobial-susceptibility testing. A combination of a broad-spectrum $\beta$-lactam agent and an aminoglycoside is usually synergistic. $\beta$-Lactams with antipseudomonal activity include ceftazidime, cefepime, piperacillin, imipenem, meropenem, and aztreonam. Tobramycin and amikacin are the most active aminoglycosides, and ciprofloxacin has the best activity amongst fluoroquinolones. In cases of multiresistant strains, polymyxin or colistin may be necessary. Rates of failure of antibiotic therapy, catheter removal, and permanent transfer to hemodialysis are higher than most pathogens. ${ }^{49,50}$ 


\section{Acinetobacter peritonitis}

Acinetobacter peritonitis is not common, accounting for $3 \%-7 \%$ of cases in reported series. ${ }^{51,52}$ Like Pseudomonas, Acinetobacter is inherently resistant to most antibiotics and readily develops resistance through mutations and acquisition of genetic material from other organisms..$^{53}$ There is a relatively high incidence of therapeutic failure, catheter removal, and mortality with Acinetobacter peritonitis, particularly with drug-resistant strains. ${ }^{51,52}$ Like Pseudomonas, therapy should be guided by results of susceptibility testing, and therapy with two active drugs may be more efficacious. Most strains are susceptible to sulbactam, which is not available as a single agent but is in ampicillin-sulbactam. Carbapenems and aminoglycosides are usually active.$^{53}$ For multidrug-resistant organisms, tigecycline and minocycline are often active, but there is little experience using these drugs in PD peritonitis. Finally, polymyxin B and colistin are usually active. Therapy should continue for 3 weeks.

\section{Stenotrophomonas peritonitis}

Stenotrophomonas maltophilia is another nonfermenting Gram-negative rod that is usually multidrug-resistant. In published series, it generally accounts for less than 5\% of peritonitis cases. ${ }^{54,55} \mathrm{~A}$ recent course of antibiotics was found to be a risk factor in one series. ${ }^{54}$ Stenotrophomonas is highly resistant to most $\beta$-lactam antibiotics, particularly carbapenems and aminoglycosides. It has a chromosomal carbapenemase. The most active drugs are trimethoprimsulfamethoxazole, colistin, aztreonam, piperacillintazobactam, and moxifloxacin. Based on limited experience, Stenotrophomonas peritonitis should be treated with two effective drugs for 3 weeks. Despite this, there is a high rate of failure and catheter loss.

\section{Polymicrobial peritonitis}

Polymicrobial peritonitis, the isolation of more than one organism in a single peritonitis episode, is reported in about $10 \%$ of cases in most series. ${ }^{56,57}$ Polymicrobial peritonitis is more common in patients with prior episodes of peritonitis. Polymicrobial peritonitis with only Gram-positive organisms makes up about $20 \%$ of cases and has the best prognosis in terms of cure rates and catheter retention. Infections that include Gram-negative bacteria, anaerobes, or fungi have worse outcomes, with higher failure rates, catheter removal, and permanent transfer to hemodialysis. ${ }^{56-58}$ Isolation of multiple species, especially Gram-negative enteric bacteria and anaerobes should raise suspicion for an intraabdominal infection, which is present in less than $10 \%$ of cases. Antimicrobial therapy should be guided by culture and sensitivity results and continued for 3 weeks. Patients with a poor response to therapy should have their catheters removed promptly to preserve their peritoneal membrane. ${ }^{57}$

\section{Culture-negative peritonitis}

Culture-negative peritonitis, as the name implies, is a case with clinical findings of peritonitis: abdominal pain, elevated peritoneal fluid WBCs, and peritoneal fluid cultures not growing a pathogen. Potential causes of culture-negative cases are antibiotic administration prior to peritoneal fluid cultures, suboptimal handling or processing of cultures or culture techniques, ${ }^{59-61}$ or the presence of fastidious organisms, AFB, or filamentous fungi. Guidelines recommend a benchmark of less than $20 \%$ culture-negative cases. Generally, patients with culture-negative peritonitis do well. Peritoneal fluid should be reevaluated at day 3 to assess response to therapy. ${ }^{2}$ Those who respond to initial empiric therapy can have their Gramnegative antibiotic discontinued, and can be treated with 2 weeks of vancomycin or cefazolin. ${ }^{2,59}$ Patients who do not respond to empiric therapy with a reduction in abdominal pain and/or improvement in peritoneal WBC count need to be reevaluated for infection with fastidious bacteria, fungi, or mycobacterial infection with more involved bacterial cultures and cultures for AFB and fungi. A lack of response should also prompt consideration for catheter removal after 5-7 days.

\section{Fungal peritonitis}

The incidence of fungal peritonitis varies from center to center, from $<5 \%$ to $15 \%$ of cases. ${ }^{62}$ Candida spp. account for $90 \%$ or more of episodes of fungal peritonitis. In older studies, the majority of Candida peritonitis cases were caused by Candida albicans. More recent series have reported Candida albicans in less than half their cases, with frequent isolation of Candida parapsilosis, Candida tropicalis, and Candida krusei ${ }^{62-65}$ Candida spp. are part of the normal flora in most people's skin and GI and GU tracts, and antibiotic therapy increases their density of colonization. Therefore, most cases of Candida peritonitis follow a recent course of antibiotics for peritonitis or other indications. Touch contamination is the usual route of infection.

The clinical presentation of Candida peritonitis resembles bacterial peritonitis, with abdominal pain and elevated peritoneal fluid WBC count with neutrophilic predominance. Candida exists only in yeast form, and grows readily on routine bacteria-culture media. Specific fungal cultures are unnecessary. Antifungal drug-susceptibility testing is widely available, and tests for fluconazole, flucytosine, amphotericin B, 
and caspofungin. In cases of Candida peritonitis, initial empiric therapy should be with fluconazole, pending species identification and drug-susceptibility testing. Fluconazole is administered at $200 \mathrm{mg}$ intraperitoneally every $24-48$ hours or given orally at a loading dose of $200 \mathrm{mg}$ followed by 50-100 mg daily. Drug-susceptibility testing should be obtained, because $C$. krusei is always resistant to fluconazole and Candida glabrata often resistant. In addition, other Candida spp. may become resistant. ${ }^{65}$ In the event of fluconazole-resistant organisms, therapy with amphotericin B $0.75-1 \mathrm{mg} / \mathrm{kg} /$ day IV along with oral flucytosine $1 \mathrm{~g}$ daily has been used successfully. Intraperitoneal amphotericin is very irritating and difficult to use. If flucytosine is used, trough serum levels should be measured with a target level of $25-50 \mu \mathrm{g} / \mathrm{mL}$ to avoid toxicity. ${ }^{66}$ Voriconazole is a newer triazole antifungal that is active on many fluconazole-resistant strains. ${ }^{67}$ There is little experience using voriconazole to treat Candida peritonitis, but it has been used successfully in the treatment of peritonitis caused by filamentous fungi at a dose of $200 \mathrm{mg}$ intraperitoneally once daily or $200 \mathrm{mg}$ orally twice a day. ${ }^{68,69}$

Candida peritonitis is difficult to treat effectively, and results in a high rate of catheter loss and permanent transfer to hemodialysis. ISPD guidelines and a number of other sources recommend immediate catheter removal upon diagnosis of Candida peritonitis, followed by 2 weeks of antifungal therapy. ${ }^{2,62,64,65,70}$ However, two published series showed fairly good success with other treatment modalities. Wang et al treated 13 Candida peritonitis patients with IV amphotericin B and oral fluconazole, leaving the catheter in place until the effluent cleared.$^{66}$ These patients were treated with antifungals for 1-2 more weeks, followed by catheter replacement about 4 weeks after the onset of treatment. However, of these 13 patients, none successfully returned to PD. Boer et al successfully treated eight Candida peritonitis patients with catheters in place. ${ }^{71}$ Patients received fluconazole $150 \mathrm{mg}$ intraperitoneally every 48 hours, oral flucytosine $500 \mathrm{mg}$ twice daily, and catheters were locked with $10 \mathrm{~mL}$ amphotericin B $0.1 \mathrm{mg} / \mathrm{mL}$ after each continuous ambulatory PD exchange.

The majority of patients with Candida peritonitis have received a recent course of antibiotics for peritonitis or other indications prior to the diagnosis of Candida peritonitis. $^{72}$ Broad-spectrum antibiotics reduce the normal bacterial flora of the skin and GI and GU tracts, and facilitate overgrowth with Candida spp. Early studies suggested that therapy with oral nystatin, a nonabsorbable antifungal, taken during antibiotic therapy might reduce the incidence of secondary fungal peritonitis, but the degree of protection was not large. ${ }^{73}$ A more recent randomized controlled trial in which patients who were receiving antibiotics for bacterial peritonitis, administration of fluconazole $200 \mathrm{mg}$ orally every 48 hours reduced the incidence of secondary fungal peritonitis by more than $80 \%$. ${ }^{74}$ This is now recommended in the ISPD guidelines. ${ }^{2}$

Peritonitis caused by filamentous fungi or molds is less common than Candida, accounting for less than $10 \%$ of cases of fungal peritonitis. ${ }^{72}$ These organisms are ubiquitous in the environment, and probably cause peritonitis via touch contamination. Clinical presentation and peritoneal fluid studies resemble bacterial peritonitis. ${ }^{75}$ These organisms do not grow well on standard bacteria-culture medias, so in culture-negative cases that fail to improve by day 3 of empiric antibiotic therapy, fluid should be obtained for fungal cultures. These cultures are inoculated on fungal-culture media, and the organisms grow as molds. The most frequently reported organisms are Aspergillus, followed by Mucor (mucormycosis), but a variety of other species have been reported to cause fungal peritonitis. ${ }^{62,75}$ Upon diagnosis, the catheter should be removed promptly. These organisms are almost always resistant to fluconazole, but may be susceptible to voriconazole or posaconazole and are usually susceptible to amphotericin B. There is not a lot of published experience with these infections, but most would treat with effective antifungal therapy for 2-8 weeks before attempting catheter replacement. Case reports have had success with intraperitoneal and oral voriconazole for Aspergillus peritonitis and liposomal amphotericin B and oral posaconazole for Mucor peritonitis. ${ }^{68,69}$ Despite optimal management, half or more of these patients will experience technique failure and transfer to hemodialysis. ${ }^{76,77}$

\section{Mycobacterial peritonitis}

Human mycobacterial infections are lumped into two groups: those caused by Mycobacterium tuberculosis (MTB) and those caused by non-tuberculous mycobacteria (NTM). MTB infection is spread from one person to another, usually establishing a latent infection that may reactivate. NTM are environmental organisms that do not spread from person to person, and although uncommon causes of $\mathrm{PD}$ peritonitis, seem to be increasing in incidence.

\section{Tuberculous peritonitis}

Tuberculous peritonitis usually arises as reactivation of a latent tuberculous infection. Chronic renal insufficiency is immunosuppressive and associated with a 100-fold increase in the incidence of reactivation disease. ${ }^{78}$ It should be routine practice to screen patients for latent tuberculous infection as they prepare for dialysis or enter a dialysis program, 
especially if they live in or have emigrated from an area that has a high incidence of latent tuberculous infection (Asia, Africa, South or Central America). This is easily done with a tuberculin skin test or an IFN $\gamma$-release assay. ${ }^{79}$ Patients who are diagnosed with a latent tuberculous infection should receive preventive therapy with isoniazid $300 \mathrm{mg}$ daily or $900 \mathrm{mg}$ three times a week with pyridoxine $50 \mathrm{mg}$ daily for 9 months. This will greatly reduce their risk of developing active TB.

Of PD patients who develop active TB, peritonitis is the site of infection (37\%) nearly as often as pulmonary TB $(40 \%) .{ }^{80}$ The clinical presentation of tuberculous peritonitis resembles bacterial infection, with fever, abdominal pain, and cloudy dialysate with elevated WBCs that are usually predominantly PMNLs. ${ }^{81,82}$ The main difference is TB peritonitis tends to have more subacute onset of signs and symptoms. Routine bacterial cultures of peritoneal fluid are negative and AFB smears of fluid are rarely positive. At the time of presentation, TB skin tests are usually negative, due to anergy. ${ }^{83,84}$ In cases of culture-negative peritonitis, TB should be considered and fluid sent for AFB smears and cultures. The rate of positive AFB smears of peritoneal fluid is less than $20 \%$ in most series, and AFB cultures using the BacT system are positive in 70\%-90\%, but take 10-14 days to become positive. Peritoneal biopsy is usually positive with granulomas present and positive cultures, but is invasive. A more rapid means of diagnosis is a direct PCR of PD fluid. PCR for MTB yields results in a few hours, and is very sensitive and specific for TB peritonitis. ${ }^{81,83-85}$

Upon diagnosis of TB peritonitis, four-drug antituberculous therapy should be started. ISPD guidelines recommend isoniazid $300 \mathrm{mg}$ once daily, rifampin $600 \mathrm{mg}$ once daily, pyrazinamide $25-35 \mathrm{mg} / \mathrm{kg}$ three times weekly, and oral ofloxacin $200 \mathrm{mg}$ once daily, with oral pyridoxine $50 \mathrm{mg}$ once weekly. ${ }^{2}$ Ethambutol is not recommended, because of the risk of optic neuritis. With drug-susceptible strains, pyrazinamide and ofloxacin may be stopped after 2 months and isoniazid and rifampin continued for a total of 12-18 months. In several series, treatment without catheter removal resulted in good outcomes. ${ }^{80,82-84}$ Despite optimal diagnosis and therapy, mortality rates are $20 \%-30 \%$.

\section{Nontuberculous mycobacterial peritonitis}

NTM are a group of 150 or more species, many of which have been identified as human pathogens. These organisms exist in the environment in water, soil, animals, and birds, are usually acquired from water or other environmental sources, and are not transmitted from one person to another. ${ }^{86}$ In most centers in the USA, peritonitis caused by NTM is more common than MTB. NTM may also cause exit-site infections, which may lead to peritonitis. Use of gentamicin for exit-site prophylaxis has been associated with an increased incidence of NTM exit-site infection. ${ }^{6,87}$

The clinical presentation of NTM peritonitis resembles bacterial peritonitis, with abdominal pain and cloudy effluent with a neutrophil predominance, but bacterial cultures are usually negative at 3 days. AFB smears of the fluid are usually negative. The most frequent causative organisms are the rapidly growing Mycobacterium abscessus, Mycobacterium chelonae, and Mycobacterium fortuitum..$^{88-92}$ These organisms will often grow on routine bacteria-culture media within 5 days. They are Gram-positive rods on Gram staining, and may be misidentified as diphtheroids. If that occurs, request the lab to perform an AFB stain on the isolate. Species identification and antimicrobial-susceptibility testing are necessary for effective therapy. These organisms are usually resistant to standard antituberculous drugs like isoniazid, rifampin, and pyrazinamide. Treatment should be guided by antimicrobial drug-susceptibility testing, and often includes aminoglycosides, macrolides, and fluoroquinolones. Over $80 \%$ of reported cases require catheter removal with antimicrobial therapy continued for at least 6 weeks thereafter.

\section{Preventing peritonitis}

Rates of PD-associated peritonitis have decreased substantially over the years through improvements in equipment, techniques, and prophylactic measures. The 2016 ISPD guidelines recommended a benchmark of 0.5 episodes per year or one episode every 2 years. ${ }^{2}$ Reduction in peritonitis cases is a multifaceted process, starting with extensive patient training, focusing on proper technique..$^{93,94}$ The use of two-bag, flush-before-fill with Y connectors is thought to be partially responsible for reducing peritonitis rates. Administration of prophylactic antibiotics with vancomycin or first- or second-generation cephalosporins prior to catheter placement has been shown to reduce postoperative catheter infections and is recommended in ISPD guidelines. ${ }^{2,95}$ Some evidence suggests that screening patients for nasal carriage of $S$. aureus and treating carriers with intranasal mupirocin reduces postoperative Staphylococcus infections. ${ }^{6,95}$ The choice between double-cuff, swan-neck, or Tenckhoff catheters seems to have little effect on peritonitis rates, nor does choice of dialysis fluids. ${ }^{2}$

In the event of a break in technique, like touch contamination, the patient should be educated to notify their provider 
immediately. If fluid has not been infused, the patient may need to have the end of the tubing exchanged. If it is likely contamination has occurred and fluid has been infused, a short course of prophylactic antibiotics is probably indicated, depending on the nature of the contamination. This usually consists of a single intraperitoneal dose of cefazolin or a 2-day course of an oral antibiotic, such as cephalexin. ${ }^{95}$

Patients should be trained in proper exit-site care. Good hand hygiene prior to any exit-site care is very important. The exit site should be cleansed at least twice a week and after showering with antibacterial soap and water or chlorhexidine soaps. ${ }^{2,6}$ Daily application of antimicrobials to the exit site has been shown to reduce exit-site infections and peritonitis. Mupirocin cream applied daily to the exit site has been shown to reduce $S$. aureus exit-site infections and peritonitis by $73 \% .{ }^{96}$

Mupirocin only has activity against Staphylococcus, Streptococcus, and other Gram-positive bacteria. Gentamicin cream is also effective in reducing exit-site infections caused by $S$. aureus, Pseudomonas, and other Gram-negative bacteria. ${ }^{97}$ However, some studies have reported increased incidence of exit-site infections with $S$. aureus, Gram-negative enteric bacteria, Pseudomonas, ${ }^{98}$ and NTM in patients using gentamicin for exit-site prophylaxis. ${ }^{6,87}$

Invasive diagnostic and therapeutic procedures that result in transient bacteremia have been implicated as causes of peritonitis. Antibiotic prophylaxis with a single $2 \mathrm{~g}$ oral dose of amoxicillin 2 hours prior to dental procedures that induce bleeding of the gums is recommended. ${ }^{95}$ Periprocedural antibiotic prophylaxis with parenteral ampicillin and gentamicin is also recommended for patients undergoing colonoscopy, hysteroscopy, or other invasive procedures that disrupt colonized mucosal surfaces to prevent peritonitis. ${ }^{2,95}$ As mentioned earlier, antifungal prophylaxis with oral fluconazole is recommended for $\mathrm{PD}$ patients who are receiving antibiotics for peritonitis or other infections. ${ }^{2}$

\section{Disclosure}

The author reports no conflicts of interest in this work.

\section{References}

1. Grassmann A, Gioberges S, Moeller S, Brown G. ESRD patients in 2004: global overview of patient numbers, treatment modalities and associated trends. Nephrol Dial Transplant. 2005;20:2587-2593.

2. Li PK, Szeto CC, Piraino B, et al. ISPD peritonitis recommendations: 2016 update on prevention and treatment. Perit Dial Int. 2016;36:481-508.

3. Mujais S. Microbiology and outcomes of peritonitis in North America. Kidney Int. 2006;70:S55-S62.

4. Ghali JR, Bannister KM, Brown FG, et al. Microbiology and outcomes of peritonitis in Australian peritoneal dialysis patients. Perit Dial Int. 2011;31:651-662.
5. van Duepen AT, Tomlinson GA, Jassal SV. The association between exit site infection and subsequent peritonitis among peritoneal dialysis patients. Clin J Am Soc Nephrol. 2012;7:1266-1271.

6. Szeto CC, Li PK, Johnson DW, et al. ISPD catheter-related infection recommendations: 2017 update. Perit Dial Int. 2017;37:141-154.

7. Burkhalter F, Clemenger M, Haddoub SS, McGrory J, Hisole N, Brown E. Pseudomonas exit site infection: treatment outcomes with topical gentamicin in addition to systemic antibiotics. Clin Kidney J. 2015;8:781-784.

8. Alfa MA, Degagne P, Olson N, Harding GK. Improved detection of bacterial growth in continuous ambulatory peritoneal dialysis effluent by use of BacT/Alert FAN bottles. J Clin Microbiol. 1997;35:862-866.

9. Beiber SD, Anderson AE, Mehrota R. Diagnostic testing for peritonitis in patients undergoing peritoneal dialysis. Semin Dial. 2014;27:602-606.

10. Ruger W, van Ittersum FJ, Comazzettos SE, Hoeks SE, ter Wee PM. Similar peritonitis outcomes in CAPD and APD patients with dialysis modality continuation during peritonitis. Perit Dial Int. 2011;31:39-47.

11. de Moraes TP, Olandoski M, Caramori JC, et al. Novel predictors of peritonitis-related outcomes in the BRAZPD cohort. Perit Dial Int. 2012;34: 179-187.

12. von Eiff C, Peters G, Heilmann C. Pathogenesis of infections due to coagulase-negative staphylococci. Lancet Infect Dis. 2002;2:677-685.

13. Donlan RM. Biofilm formation: a clinically relevant microbiologic process. Clin Infect Dis. 2001;33:1387-1392.

14. Burke M, Hawley CM, Badve SV, et al. Relapsing and recurrent peritoneal dialysis-associated peritonitis: a multicenter registry study. $\mathrm{Am}$ J Kidney Dis. 2011;58:429-436.

15. Fahim M, Hawley CM, McDonald SP, et al. Coagulase-negative staphylococcal peritonitis in Australian peritoneal dialysis patients: predictors, treatment, and outcome in 936 cases. Nephrol Dial Transplant. 2010;25:3386-3392.

16. Camargo CH, da Cunha ML, Caramori JC, Mondelli AL, Montelli AC, Barretti P. Peritoneal dialysis-related peritonitis due to coagulasenegative Staphylococcus: a review of 115 cases in a Brazilian center. Clin J Am Soc Nephrol. 2014;9:1074-1081.

17. Luzar MA, Coles Ga, Faller B, et al. Staphylococcus aureus nasal carriage and infection in patients on continuous ambulatory peritoneal dialysis. N Engl J Med. 1990;372:505-509.

18. Ritzou J, Hoffmann RM, Tzamaloukas AH. Effect of preventing Staphylococcus aureus carriage on rates of peritoneal catheter-related staphylococcal infections. Perit Dial Int. 2001;21:471-479.

19. Szeto CC, Chow KM, Kwan BC, et al. Staphylococcus aureus complicates peritoneal dialysis: review of 245 consecutive cases. Clin J Am Soc Nephrol. 2007;2:245-251.

20. Govindarajulu S, Hawley CM, McDonald SP, et al. Staphylococcus aureus peritonitis in Australian peritoneal dialysis patients: predictors, treatment and outcomes in 503 patients. Perit Dial Int. 2010;30: 311-319.

21. Khadzhynov D, Joukhadar C, Peters H. Plasma and peritoneal dialysate levels during daptomycin therapy for peritonitis. Am J Kidney Dis. 2009;53:911-912.

22. Bahte SK, Bertram A, Burkhardt O, et al. Therapeutic serum concentrations of daptomycin after intraperitoneal administration in a patient with peritoneal dialysis associated peritonitis. JAntimicrob Chemother. 2010;65:1312-1314.

23. Saint-Paul LP, Ficheux M, Debruyn D, et al. Pharmacokinetics of intraperitoneal daptomycin in patients with peritoneal dialysis-related peritonitis. Perit Dial Int. 2017;37:44-50.

24. Song IJ, Seo JW, Kwon YE, et al. Successful treatment of vancomycinresistant enterococcus peritonitis using linezolid without catheter removal in a peritoneal dialysis patient. Perit Dial Int. 2014;34:235-239.

25. Gervasoni C, Bergia R, Cozzi V, Clementi E, Cattaneo D. Is it time to revise linezolid doses in peritoneal dialysis patients? J Antimicrob Chemother. 2015;70:2918-2920.

26. Saravolatz LD, Stein GE, Johnson LB. Ceftaroline: a novel cephalosporin with activity against methicillin-resistant Staphylococcus aureus. Clin Infect Dis. 2011;52:1156-1163. 
27. O'Shea S, Hawley CM, McDonald SP, et al. Streptococcal peritonitis in Australian peritoneal dialysis patients: predictors, treatment and outcomes in 287 cases. BMC Nephrol. 2009;10:19.

28. Shukla A, Abreu Z, Bargman JM. Streptococcal PD peritonitis: a 10-year review of one centre's experience. Nephrol Dial Transplant. 2006;21: 3545-3549.

29. Hsu RB, Fang-Yue L. Effect of penicillin resistance on presentation and outcome of non-enterococcal streptococcal infective endocarditis. Cardiology. 2006;105:234-239.

30. Roberts DM, Fernando G, Singer RF, Kennedy KJ, Lawrence M, Talaulikar G. Antibiotic stability in commercial peritoneal dialysis solutions: influence of formulation, storage, and duration. Nephrol Dial Transplant. 2011;26:3344-3349.

31. Chao CJ, Lee SY, Yang WS, et al. Viridans streptococci in peritoneal dialysis peritonitis: clinical courses and long-term outcomes. Perit Dial Int. 2015;35:333-341.

32. Edey M, Hawley CM, McDonald SP, et al. Enterococcal peritonitis in Australian peritoneal dialysis patients: predictors, treatment, and outcomes in 116 cases. Nephrol Dial Transplant. 2010;25:1272-1278.

33. Yip T, Tsa KC, Ng F, et al. Clinical course and outcomes of single organism enterococcus peritonitis in peritoneal dialysis patients. Perit Dial Int. 2011;31:522-528.

34. Huen SC, Hall I, Topal J, Mahnensmith RL, Brewster UC, Abu-Alfa AK. Successful use of intraperitoneal daptomycin in the treatment of vancomycin-resistant enterococcus peritonitis. Am J Kidney Dis 2009;54:538-541.

35. Gilmore JF, Kin M, LaSalvia MT, Mahoney MV. Treatment of enterococcal peritonitis with intraperitoneal daptomycin in a vancomycin-allergic patient and a review of the literature. Perit Dial Int. 2013;33:353-357.

36. Yang JW, Kim YS, Choi SO, Han BG. Successful use of intravenous linezolid in CAPD patient with vancomycin-resistant enterococcal peritonitis. Perit Dial Int. 2011;31:209-210.

37. Wu VC, Wang YT, Wang CY, et al. High frequency of linezolidassociated thrombocytopenia and anemia among patients with end-stage renal disease. Clin Infect Dis. 2006;42:66-72.

38. Song IJ, Seo JW, Kwon YE, et al. Successful treatment of vancomycinresistant enterococcus peritonitis using linezolid without catheter removal in a peritoneal dialysis patient. Perit Dial Int. 2014;34:235-239.

39. Barraclough K, Hawley CM, McDonald SP, et al. Corynebacterium peritonitis in Australian peritoneal dialysis patients: predictors, treatment and outcomes in 82 cases. Nephrol Dial Transplant. 2009;24:3834-3839.

40. Szeto CC, Chow KM, Chung KY, Kwan BC, Leung CB, Li PK. The clinical course of peritoneal dialysis-related peritonitis caused by Corynebacterium species. Nephrol Dial Transplant. 2005;20: 2793-2796.

41. Jain AK, Blake PG. Non-Pseudomonas Gram-negative peritonitis. Kidney Int. 2006;69:1107-1109.

42. Szeto CC, Chow VC, Chow KM, et al. Enterobacteriaceae peritonitis complicating peritoneal dialysis: a review of 210 consecutive cases. Kidney Int. 2006;69:1245-1252.

43. Jarvis EM, Hawley CM, McDonald SP, et al. Predictors, treatment and outcomes of non-Pseudomonas Gram negative peritonitis. Kidney Int. 2010;78:408-414.

44. Bradford PA. Extended-spectrum $\beta$-lactamases in the 21 st century: characterization, epidemiology, and detection of this important resistance threat. Clin Microbiol Rev. 2001;14:933-951.

45. Wong SS, Ho PL, Yuen KY. Evolution of antibiotic resistance mechanisms and their relevance to dialysis-related infections. Perit Dial Int. 2007;27:S272-S280.

46. Yip T, Tse KC, Lam MF, et al. Risk factors and outcomes of extendedspectrum $\beta$-lactamase producing $E$. coli peritonitis in CAPD patients. Perit Dial Int. 2006;26:191-197.

47. Feng X, Yang X, Yi C, et al. Escherichia coli peritonitis in peritoneal dialysis: the prevalence, antibiotic resistance, and clinical outcomes in a South China dialysis center. Perit Dial Int. 2014:308-316.
48. Gupta N, Limbago BM, Patel JB, Kallen AJ. Carbapenem-resistant Enterobacteriaceae: epidemiology and prevention. Clin Infect Dis. 2011;53:60-67.

49. Szeto CC, Chow KM, Leung CB, et al. Clinical course of peritonitis due to Pseudomonas species complicating peritoneal dialysis: a review of 104 cases. Kidney Int. 2001;59:2309-2315.

50. Siva B, Hawley CM, McDonald SP, et al. Pseudomonas peritonitis in Australia: predictors, treatment and outcomes in 191 cases. Clin J Am Soc Nephrol. 2009;4:957-964.

51. Li PH, Cheng VC, Yip T, Yap DY, Lui SL, Lo WK. Epidemiology and clinical characteristics of Acinetobacter peritoneal dialysis-related peritonitis in Hong Kong: with a perspective on multidrug and carbapenem resistance. Perit Dial Int. 2017;37:37:177-182.

52. Zhang W, Wu YG, Qi XM, Dai H, Lu W, Zhao M. Peritoneal dialysisrelated peritonitis with Acinetobacter baumannii: a review of seven cases. Perit Dial Int. 2012;34:317-321.

53. Munoz-Price LS, Weinstein RA. Acinetobacter infection. $N$ Engl J Med. 2008;558:1271-1281.

54. Taylor G, McKenzie M, Buchanan-Chell M, Perry D, Chui L, Dasgupta M. Peritonitis due to Stenotrophomonas maltophilia in patients undergoing chromic peritoneal dialysis. Perit Dial Int. 1999;19: 259-262.

55. Szeto CC, Li PK, Leung CB, Yu AW, Lui SF, Lai KN. Xanthomonas maltophilia peritonitis in uremic patients receiving continuous ambulatory peritoneal dialysis. Am J Kidney Dis. 1997;29:91-95.

56. Kim GC, Korbet SM. Polymicrobial peritonitis in continuous ambulatory peritoneal dialysis patients. Am J Kidney Dis. 2000;36:1000-1008.

57. Szeto CC, Chow KM, Wong TY, et al. Conservative management of polymicrobial peritonitis complicating peritoneal dialysis: a series of 140 consecutive cases. Am J Med. 2002;113:728-733.

58. Barraclough K, Hawley CM, McDonald SP, et al. Polymicrobial peritonitis in peritoneal dialysis patients in Australia: predictors, treatment, and outcomes. Am J Kidney Dis. 2010;55:121-131.

59. Fahim M, Hawley CM, McDonald SP, et al. Culture-negative peritonitis in peritoneal dialysis patients in Australia: predictors, treatment and outcomes in 435 patients. Am J Kidney Dis. 2010;55:690-697.

60. Szeto CC, Wong TY, Chow KM, Leung CB, Li PK. The clinical course of culture-negative peritonitis complicating peritoneal dialysis. Am J Kidney Dis. 2003;25:207-222.

61. Odudu A, Turner J, Coomer K, Salmon L, Yazdani F, Leung J. United Kingdom survey of culture-negative peritonitis and dialysis sampling practice. Perit Dial Int. 2016;36:101-104.

62. Prasad N, Gupta A. Fungal peritonitis in peritoneal dialysis patients. Perit Dial Int. 2005;25:207-222.

63. Miles R, Hawley CM, McDonald SP, et al. Predictors and outcomes of fungal peritonitis in peritoneal dialysis patients. Kidney Int. 2009;76: 622-628.

64. Nadeau-Fredette AC, Bargman JM. Characteristics and outcomes of fungal peritonitis in a modern North American cohort. Perit Dial Int. 2015;35:78-84.

65. Levallois J, Nadeau-Fredette AC, Labbé AC, Laverdière M, Ouimet $\mathrm{D}$, Vallée $\mathrm{M}$. Ten-year experience with fungal peritonitis in peritoneal dialysis patients: antifungal susceptibility patterns in a North-American center. Int J Infect Dis. 2012;16:e41-e43.

66. Wang PN, Lo KY, Tong GM, et al. Treatment of fungal peritonitis with a combination of intravenous amphotericin B and oral flucytosine, and delayed catheter replacement in continuous ambulatory peritoneal dialysis. Perit Dial Int. 2008;28:155-162.

67. Johnson LB, Kauffman CA. Voriconazole: a new triazole antifungal agent. Clin Infect Dis. 2003;33:92-99.

68. Roberts DM, Kauter G, Ray JE, Gillin AG. Intraperitoneal voriconazole in a patient with Aspergillus peritoneal dialysis peritonitis. Perit Dial Int. 2013;33:92-93.

69. Ulusoy S, Ozkan G, Tosun I, et al. Peritonitis due to Aspergillus nidulans and its effective treatment with voriconazole: the first case report. Perit Dial Int. 2011;31:212-213. 
70. Chang TI, Kim HW, Park JT, et al. Early catheter removal improves patient survival in peritoneal dialysis patients with fungal peritonitis: results of ninety-four episodes of fungal peritonitis at a single center. Perit Dial Int. 2011;31:60-66.

71. Boer WH, van Ampting JM, Vos P. Successful treatment of eight episodes of Candida peritonitis without catheter removal using intracatheter administration of amphotericin B. Perit Dial Int. 2007;27: 208-210.

72. Matuszkiewicz-Rowinska J. Update on fungal peritonitis and its treatment. Perit Dial Int. 2009;29:S161-S165.

73. Wong PN, Lo KY, Tong GM, et al. Preventing fungal peritonitis with nystatin prophylaxis in patients receiving CAPD. Perit Dial Int. 2007;27: 531-536.

74. Restrepo C, Chacon J, Manjarres G. Fungal peritonitis in peritoneal dialysis patients: successful prophylaxis with fluconazole as demonstrated by prospective randomized control trial. Perit Dial Int. 2010;30:619-625.

75. Baer RA, Killen JP, Cho Y, Mantha M. Non-candidal fungal peritonitis in far North Queensland: a case series. Perit Dial Int. 2013;33:559-564.

76. Sedlacek M, Cotter JG, Suriawinata AA, et al. Mucormycosis peritonitis: more than 2 years of disease-free follow-up after posaconazole salvage therapy after failure of liposomal amphotericin B. Am J Kidney Dis. 2008;51:302-306.

77. Serna JH, Wanger A, Dosekun AK. Successful treatment of mucormycosis peritonitis with liposomal amphotericin B in a patient on long-term peritoneal dialysis. Am J Kidney Dis. 2003;42:E14-E17.

78. Moore DA, Lightstone L, Javid B, Friedland JS. High rates of tuberculosis in end stage renal failure: the impact of international migration. Emerg Infect Dis. 2002;8:77-78.

79. Lewinsohn DM, Leonard MK, LoBue PA, et al. Official American Thoracic Society/Infectious Disease Society of America/Centers for Disease Control and Prevention clinical practice guidelines: diagnosis of tuberculosis in adults and children. Clin Infect Dis. 2017;64:e1-e33.

80. Lui SL, Tang S, Fuk L, et al. Tuberculosis infection in Chinese patients undergoing continuous peritoneal dialysis. Am J Kidney Dis. 2001;38:1055-1060.

81. Chau TN, Leung VK, Wong S, et al. Diagnostic challenges of tuberculosis in patients with and without end-stage renal disease. Clin Infect Dis. 2007;45:e141-e146.

82. Talwani R, Horvath JA. Tuberculous peritonitis in patients undergoing continuous peritoneal dialysis: case report and review. Clin Infect Dis. 2000;31:70-75.

83. Akpolat T. Tuberculous peritonitis. Perit Dial Int. 2009;29:S166-S169.
84. Abraham G, Matthews M, Sekar L, Srikanth A, Sekar U, Soundarajan P. Tuberculous peritonitis in a cohort of continuous ambulatory dialysis patients. Perit Dial Int. 2001;21:S202-S204.

85. Edwards S, Glynn P, David MD, Kamesh L. Diagnosing tuberculous peritonitis early in patients on peritoneal dialysis: use of Xpert MTB/ RIF assay. Perit Dial Int. 2016;36:481-483.

86. Griffith DE, Askamit T, Brown-Elliott BA, et al. An official ATS/IDSA statement: diagnosis, treatment and presentation of nontuberculous mycobacterial diseases. Am J Respir Crit Care Med. 2007;175:367-416.

87. Tse KC, Lui SL, Cheng VC, et al. A cluster of rapidly growing mycobacterial peritoneal dialysis catheter exit-site infections. Am J Kidney Dis. 2007;50: E1-E5.

88. Rho M, Bia F, Brewster UC. Nontuberculous mycobacterial peritonitis in peritoneal dialysis patients. Semin Dial. 2006;20:271-276.

89. Renaud CJ, Subramanian S, Tambyah PA, Lee EJ. The clinical course of rapidly growing nontuberculous mycobacterial peritoneal dialysis infections in Asians: a case series and literature review. Nephrology (Carlton). 2011;16:174-179.

90. Song Y, Wu J, Yan H, Chen J. Peritoneal dialysis-associated nontuberculous mycobacterium peritonitis: a systematic review of reported cases. Nephrol Dial Transplant. 2012;27:1639-1644.

91. Jiang SH, Roberts DM, Clayton PA, Jardine M. Non-tuberculous mycobacterial PD peritonitis in Australia. Int Urol Nephrol. 2013;45:1423-1428.

92. Kunin M, Knecht A, Holtzman EJ. Mycobacterium chelonae peritonitis in peritoneal dialysis: literature review. Eur J Clin Microbiol Infect Dis. 2014;33:1267-1271.

93. Figueiredo AE, Bernardini J, Bowes E, et al. A syllabus for teaching peritoneal dialysis patients and caregivers. Perit Dial Int. 2016, 36:592-605.

94. Piraino B, Bernardini J, Brown E, et al. ISPD position statement on reducing the risks of peritoneal dialysis-related infections. Perit Dial Int. 2011;31:614-630.

95. Strippoli GF, Tong A, Johnson D, Schena FP, Craig JC. Antimicrobial agents to prevent peritonitis in peritoneal dialysis: a systematic review of randomized controlled trials. Am J Kidney Dis. 2004;44:591-603.

96. Xu G, Tu W, Xu C. Mupirocin for preventing exit site infection and peritonitis in patients undergoing peritoneal dialysis. Nephrol Dial Transplant. 2009;25:587-592.

97. Chen SS, Sheeth H, Piraino B, Bender F. Long-term exit-site gentamicin prophylaxis and gentamicin resistance in a peritoneal dialysis program. Perit Dial Int. 2016;36:387-389.

98. Pierce DA, Williamson JC, Mauck VS, Russell GB, Palavecino E, Burkart JM. The effect on peritoneal dialysis pathogens of changing topical antibiotic prophylaxis. Perit Dial Int. 2011;32:525-530.

\section{Publish your work in this journal}

The International Journal of Nephrology and Renovascular Disease is an international, peer-reviewed open access journal focusing on the pathophysiology of the kidney and vascular supply. Epidemiology, screening, diagnosis, and treatment interventions are covered as well as basic science, biochemical and immunological studies. The manuscript management system is completely online and includes a very quick and fair peer-review system, which is all easy to use. Visit http://www. dovepress.com/testimonials.php to read real quotes from published authors. 